\title{
Synthesis, Spectral Studies and Anti-Inflammatory Activity of 2-Acetyl Thiophene
}

\author{
B. RAMESH* ${ }^{*}$ and B. SOMESWARA RAO \\ Department of Pharmaceutical Chemistry, Sree Siddaganga College of Pharmacy, \\ B.H.Road, Tumkur-572102. Karnataka, India. \\ rameshbbatta@gmail.com
}

Received 19 August 2009; Accepted 10 October 2009

\begin{abstract}
Some new chalcones have been synthesized by the condensation of 2 -acetyl thiophene with various aromatic aldehydes in $40 \%$ alkali. The synthesized compounds were identified by spectral data and screened for antiinflammatory activity. Some of these compounds showed moderate to considerable anti-inflammatory activity.
\end{abstract}

Keywords: Chalcone, Synthesis, Anti-inflammatory activity.

\section{Introduction}

Chalcones display interesting biological activities, including antimalarial ${ }^{1}$, antiinflammatory $^{2}$, cytotoxic ${ }^{3,4}$, anticancer ${ }^{5,6}$ and antimicrobial activities ${ }^{7,8}$. In the present study, some new chalcones (1-8) have been synthesized by the reaction of 2-acetyl thiophene with different aromatic aldehydes. The structures of the various synthesized compounds are assigned on the basis of elemental analyses, IR and ${ }^{1} \mathrm{H}$ NMR spectral data. These compounds were also screened for their anti-inflammatory activity.

\section{Experimental}

Melting points were determined on a capillary melting point apparatus and are uncorrected. ${ }^{1} \mathrm{H}$ NMR spectra was recorded in the indicated solvent on Bruker WM $400 \mathrm{MHz}$ spectrometer with TMS as internal standard. Infrared spectra were recorded in $\mathrm{KBr}$ on Perkin-Elmer AC-1 spectrophotometer. Microanalyses were performed on Carlo Erba EA1108 element analyzer and were within the $\pm 0.5 \%$ of the theoretical values. Column chromatography was performed on silica gel (Merck, 60-120 mesh).

General procedure for the preparation of chalcones (1-8)

A mixture of 2-acetyl thiophene $(0.01 \mathrm{~mol})$ and appropriate aldehyde $(0.01 \mathrm{~mol})$ was stirred in ethanol $(30 \mathrm{~mL})$ and then an aqueous solution of $\mathrm{KOH}(40 \%, 15 \mathrm{~mL})$ was added to it. 
The mixture was kept overnight at room temperature and then it was poured in to crushed ice and acidified with $\mathrm{HC} 1$. The solid separated was filtered and crystallized from ethanol (Scheme 1). The characterizations data of these compounds are described in Tables $1 \& 2$.

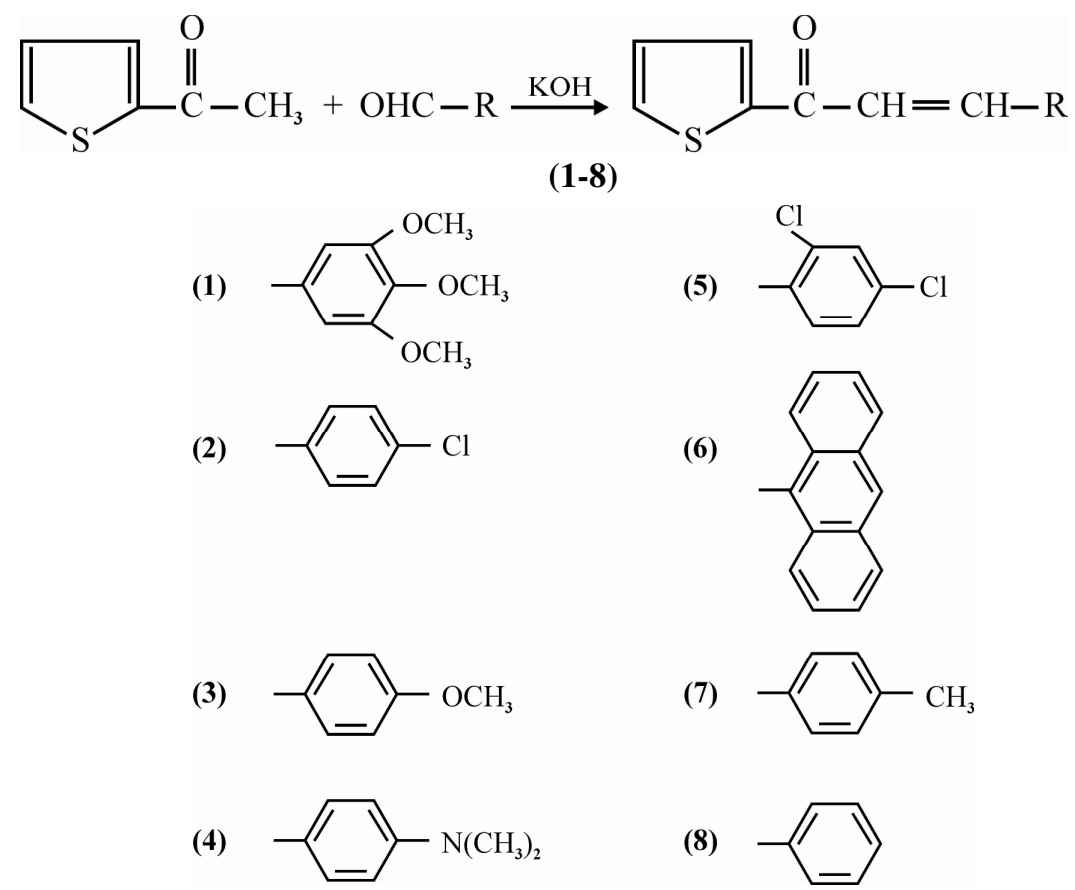

Scheme 1. Synthesis of some new chalcones of 2-acetyl thiophene (1-8).

Table 1. Physical data of compounds (1-8).

\begin{tabular}{|c|c|c|c|c|c|c|c|c|c|}
\hline \multirow{3}{*}{ 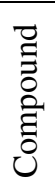 } & \multirow[b]{3}{*}{ M.F. } & \multirow{3}{*}{$\begin{array}{c}\text { M.P, } \\
{ }^{\circ} \mathrm{C}\end{array}$} & \multirow{3}{*}{$\begin{array}{c}\text { Yield, } \\
\%\end{array}$} & \multicolumn{6}{|c|}{ Elemental analyses, \% } \\
\hline & & & & \multicolumn{2}{|c|}{$\mathrm{C}$} & \multicolumn{2}{|c|}{$\mathrm{H}$} & \multicolumn{2}{|c|}{$\mathrm{N}$} \\
\hline & & & & $\begin{array}{l}\widetilde{\Xi} \\
\Xi \\
0 \\
\text { İ }\end{array}$ & $\frac{\tilde{U}}{\tilde{U}}$ & $\begin{array}{l}\vec{D} \\
\Xi \\
\text { D }\end{array}$ & $\frac{\vec{U}}{\tilde{\pi}}$ & $\begin{array}{l}\vec{D} \\
\Xi \\
0 \\
\text { İ }\end{array}$ & $\frac{\vec{U}}{\tilde{\pi}}$ \\
\hline 1 & $\mathrm{C}_{16} \mathrm{H}_{16} \mathrm{O}_{4} \mathrm{~S}$ & 135 & 95 & 43.00 & 43.20 & 43.10 & 43.20 & 10.50 & 10.80 \\
\hline 2 & $\mathrm{C}_{13} \mathrm{H}_{9} \mathrm{SCl}$ & 110 & 96 & 52.24 & 52.00 & 35.94 & 36.00 & 9.36 & 9.00 \\
\hline 3 & $\mathrm{C}_{14} \mathrm{H}_{12} \mathrm{O}_{2} \mathrm{~S}$ & 70 & 86 & 48.55 & 48.20 & 41.57 & 41.30 & 7.12 & 6.83 \\
\hline 4 & $\mathrm{C}_{15} \mathrm{H}_{15} \mathrm{OSN}$ & 100 & 83 & 45.44 & 45.45 & 45.68 & 45.45 & 3.42 & 3.03 \\
\hline 5 & $\mathrm{C}_{13} \mathrm{H}_{8} \mathrm{OSCl}_{2}$ & 95 & 92 & 51.62 & 52.00 & 32.06 & 32.00 & 3.92 & 4.00 \\
\hline 6 & $\mathrm{C}_{21} \mathrm{H}_{16} \mathrm{OS}$ & 140 & 80 & 54.90 & 53.84 & 41.11 & 41.02 & 2.59 & 2.56 \\
\hline 7 & $\mathrm{C}_{14} \mathrm{H}_{12} \mathrm{OS}$ & 105 & 89 & 50.06 & 50.00 & 42.76 & 42.85 & 3.61 & 3.51 \\
\hline 8 & $\mathrm{C}_{13} \mathrm{H}_{10} \mathrm{OS}$ & 90 & 87 & 52.09 & 52.00 & 40.11 & 40.00 & 4.06 & 4.00 \\
\hline
\end{tabular}

Anti-inflammatory activity

Synthesized compounds (1-8) were tested for their anti-inflammatory activity. Male albino rats weighing between 200-250 g were used for the experiment. Carragenan induced paw oedema method described by Singh and Ghosh ${ }^{9}$ was followed for the acute antiinflammatory model and the results are presented in Table 3. 
Table 2. Spectral data of the compounds (1-8).

\begin{tabular}{|c|c|c|}
\hline Compound. & $\mathrm{IR}\left(\mathrm{KBr}, \mathrm{cm}^{-1}\right)$ & ${ }^{1} \mathrm{H}$ NMR $\left(\mathrm{CDCl}_{3}, \mathrm{ppm}\right)$ \\
\hline 1 & $\begin{array}{l}3330(-\mathrm{OH}) \\
720(-\mathrm{C}=\mathrm{O}) \\
1650(-\mathrm{CH}=\mathrm{CH}) \\
\left.1175(-\mathrm{OCH})_{3}\right) \\
650(\mathrm{C}-\mathrm{S})\end{array}$ & $\begin{array}{l}7.69(1 \mathrm{H}, \mathrm{d}, \mathrm{J}=16 \mathrm{~Hz},-\mathrm{CO}-\mathrm{CH}=), \\
7.89(1 \mathrm{H}, \mathrm{d}, \mathrm{J}=16 \mathrm{~Hz},=\mathrm{CH}-\mathrm{Ar}) \\
3.90-3.95\left(9 \mathrm{H}, \mathrm{s}, 3 \mathrm{X}-\mathrm{OCH}_{3}\right) \\
7.20\left(1 \mathrm{H}, \mathrm{m}, \mathrm{C}-4^{1}-\mathrm{H}\right), 7.28\left(2 \mathrm{H}, \mathrm{d}, \mathrm{C}-2^{1}-\mathrm{H},\right. \\
\left.\mathrm{C}-5^{1}-\mathrm{H}\right), 7.78(2 \mathrm{H}, \mathrm{s}, \mathrm{C}-2-\mathrm{H}, \mathrm{C}-6-\mathrm{H}) .\end{array}$ \\
\hline 2 & $\begin{array}{l}1725(-\mathrm{C}=\mathrm{O}) \\
1640(-\mathrm{CH}=\mathrm{CH}) \\
850(\mathrm{C}-\mathrm{CL}) \\
650(\mathrm{C}-\mathrm{S})\end{array}$ & $\begin{array}{l}7.70(1 \mathrm{H}, \mathrm{d}, \mathrm{J}=16 \mathrm{~Hz},-\mathrm{CO}-\mathrm{CH}=), \\
7.88(1 \mathrm{H}, \mathrm{d}, \mathrm{J}=16 \mathrm{~Hz},=\mathrm{CH}-\mathrm{Ar}), \\
7.80\left(1 \mathrm{H}, \mathrm{d}, \mathrm{J}=9 \mathrm{~Hz}, \mathrm{C}-5^{1}-\mathrm{H}\right), \\
7.58(2 \mathrm{H}, \mathrm{d}, \mathrm{C}-3-\mathrm{H}, \mathrm{C}-5-\mathrm{H}), \\
7.40(2 \mathrm{H}, \mathrm{d}, \mathrm{C}-2-\mathrm{H},-\mathrm{C}-6-\mathrm{H}), \\
7.38(1 \mathrm{H}, \mathrm{d}, \mathrm{J}=16 \mathrm{~Hz}, \mathrm{C}-3-\mathrm{H}), \\
7.20\left(1 \mathrm{H}, \mathrm{m}, \mathrm{C}-4^{1}-\mathrm{H}\right) .\end{array}$ \\
\hline 3 & $\begin{array}{l}1720(-\mathrm{C}=\mathrm{O}) \\
1648(-\mathrm{CH}=\mathrm{CH}) \\
1170\left(-\mathrm{OCH}_{3}\right) \\
666(\mathrm{C}-\mathrm{S})\end{array}$ & $\begin{array}{l}6.94(1 \mathrm{H}, \mathrm{d}, \mathrm{J}=16 \mathrm{~Hz},-\mathrm{CO}-\mathrm{CH}=), \\
7.61(1 \mathrm{H}, \mathrm{d}, \mathrm{J}=16 \mathrm{~Hz}=\mathrm{CH}-\mathrm{Ar}), \\
7.86\left(2 \mathrm{H}, \mathrm{d}, \mathrm{C}-3^{1}-\mathrm{H},-\mathrm{C}-5^{1}-\mathrm{H}\right), \\
7.68(2 \mathrm{H}, \mathrm{d}, \mathrm{C}-3-\mathrm{H},-\mathrm{C}-5-\mathrm{H}), \\
7.18\left(1 \mathrm{H}, \mathrm{m}, \mathrm{C}-4^{1}-\mathrm{H}\right) \\
6.92(2 \mathrm{H}, \mathrm{d},-\mathrm{C}-2-\mathrm{H},-\mathrm{C}-6-\mathrm{H}) .\end{array}$ \\
\hline 4 & $\begin{array}{l}1730(-\mathrm{C}=0), \\
1638(-\mathrm{CH}=\mathrm{CH}), \\
1180\left(\mathrm{~N}\left(\mathrm{CH}_{3}\right)_{2}\right), \\
676(\mathrm{C}-\mathrm{S})\end{array}$ & $\begin{array}{l}6.70(1 \mathrm{H}, \mathrm{d}, \mathrm{J}=16 \mathrm{~Hz},-\mathrm{CO}-\mathrm{CH}=), 7.55(1 \mathrm{H}, \mathrm{d}, \\
\mathrm{J}=16 \mathrm{~Hz},=\mathrm{CH}-\mathrm{Ar}), 3.05\left(1 \mathrm{H}, \mathrm{s}, \mathrm{N}\left(\mathrm{CH}_{3}\right)_{2}\right), 7.86 \\
\left(2 \mathrm{H}, \mathrm{d}, \mathrm{C}-5^{1}-\mathrm{H}, \mathrm{C}-3^{1}-\mathrm{H}\right), 7.82(2 \mathrm{H}, \mathrm{d}, \mathrm{C}-3-\mathrm{H}, \\
\mathrm{C}-5-\mathrm{H}), 7.63(2 \mathrm{H}, \mathrm{d}, \mathrm{C}-2-\mathrm{H}, \mathrm{C}-6-\mathrm{H}), \\
7.17\left(1 \mathrm{H}, \mathrm{m}, \mathrm{C}-4^{1}-\mathrm{H}\right) .\end{array}$ \\
\hline 5 & $\begin{array}{l}1735(-\mathrm{C}=\mathrm{O}) \\
1636(-\mathrm{CH}=\mathrm{CH}) \\
855(\mathrm{C}-\mathrm{Cl}) \\
686(\mathrm{C}-\mathrm{S})\end{array}$ & $\begin{array}{l}7.31(1 \mathrm{H}, \mathrm{d}, \mathrm{J}=16 \mathrm{~Hz},-\mathrm{CO}-\mathrm{CH}=), \\
7.87(1 \mathrm{H}, \mathrm{d}, \mathrm{J}=16 \mathrm{~Hz},=\mathrm{CH}-\mathrm{Ar}) 7.71(1 \mathrm{H}, \mathrm{d}, \\
\mathrm{J}=8 \mathrm{~Hz},-\mathrm{C}-5-\mathrm{H}), 7.69\left(1 \mathrm{H}, \mathrm{d}, \mathrm{J}=9 \mathrm{~Hz},-\mathrm{C}-3^{1}-\mathrm{H}\right), \\
7.67(1 \mathrm{H}, \mathrm{d},-\mathrm{C}-6-\mathrm{H}), 7.48(1 \mathrm{H}, \mathrm{s},-\mathrm{C}-3-\mathrm{H}), \\
7.20\left(1 \mathrm{H}, \mathrm{m},-\mathrm{C}-4^{1}-\mathrm{H}\right) .\end{array}$ \\
\hline 6 & $\begin{array}{l}1710(-\mathrm{C}=0), \\
1655(-\mathrm{CH}=\mathrm{CH}) \\
645(\mathrm{C}-\mathrm{S})\end{array}$ & $\begin{array}{l}7.44(1 \mathrm{H}, \mathrm{d}, \mathrm{J}=16 \mathrm{~Hz},-\mathrm{CO}-\mathrm{CH}=), 7.74(1 \mathrm{H}, \mathrm{d}, \\
\mathrm{J}=16 \mathrm{~Hz},=\mathrm{CH}-\mathrm{Ar}) 8.83(1 \mathrm{H}, \mathrm{d}, \mathrm{J}=9 \mathrm{~Hz}, \\
\left.\mathrm{C}-5^{1}-\mathrm{H}\right), 8.09\left(1 \mathrm{H}, \mathrm{m}, \mathrm{C}-4^{1}-\mathrm{H}\right), 8.06(1 \mathrm{H}, \mathrm{d}, \\
\left.\mathrm{J}=9 \mathrm{~Hz}, \mathrm{C}-3^{1}-\mathrm{H}\right), 7.18-7.79(10 \mathrm{H}, \mathrm{m}, \mathrm{Ar}-\mathrm{H}) .\end{array}$ \\
\hline 7 & $\begin{array}{l}1732(-\mathrm{C}=\mathrm{O}) \\
1645(-\mathrm{CH}=\mathrm{CH}) \\
652(\mathrm{C}-\mathrm{S})\end{array}$ & $\begin{array}{l}7.39(1 \mathrm{H}, \mathrm{d}, \mathrm{J}=16 \mathrm{~Hz},-\mathrm{CO}-\mathrm{CH}=), 7.56(1 \mathrm{H}, \mathrm{d}, \\
\mathrm{J}=16 \mathrm{~Hz},=\mathrm{CH}-\mathrm{Ar}), 7.88(1 \mathrm{H}, \mathrm{d}, \mathrm{J}=9 \mathrm{~Hz} \\
\left.-\mathrm{C}-5^{1}-\mathrm{H}\right), 7.68(2 \mathrm{H}, \mathrm{d},-\mathrm{C}-2-\mathrm{H},-\mathrm{C}-6-\mathrm{H}) \\
7.58\left(1 \mathrm{H}, \mathrm{d}, \mathrm{J}=8 \mathrm{~Hz},-\mathrm{C}-3^{1}-\mathrm{H}\right), 7.23(2 \mathrm{H}, \mathrm{d} \\
-\mathrm{C}-3-\mathrm{H},-\mathrm{C}-5-\mathrm{H}), 7.19\left(1 \mathrm{H}, \mathrm{m},-\mathrm{C}-4^{1}-\mathrm{H}\right)\end{array}$ \\
\hline 8 & $\begin{array}{l}1700(-\mathrm{C}=0) \\
1650(-\mathrm{CH}=\mathrm{CH}) \\
650(\mathrm{C}-\mathrm{S})\end{array}$ & $\begin{array}{l}7.69(1 \mathrm{H}, \mathrm{d}, \mathrm{J}=16 \mathrm{~Hz},-\mathrm{CO}-\mathrm{CH}=), 7.84(1 \mathrm{H}, \mathrm{d}, \\
\mathrm{J}=16 \mathrm{~Hz}=\mathrm{CH}-\mathrm{Ar}), 7.19(1 \mathrm{H}, \mathrm{m}, \mathrm{C}-4-\mathrm{H}), 7.87 \\
\left(1 \mathrm{H}, \mathrm{d}, \mathrm{J}=9 \mathrm{~Hz}, \mathrm{C}-5^{1}-\mathrm{H}\right), 7.44\left(1 \mathrm{H}, \mathrm{m}, \mathrm{C}-4^{1}-\mathrm{H}\right) \\
7.42\left(1 \mathrm{H}, \mathrm{d}, \mathrm{J}=9 \mathrm{~Hz}, \mathrm{C}-3^{1}-\mathrm{H},\right), 7.40(2 \mathrm{H}, \mathrm{d} \\
\mathrm{C}-3-\mathrm{H}, \mathrm{C}-5-\mathrm{H}), 7.64(2 \mathrm{H}, \mathrm{m}, \mathrm{C}-2-\mathrm{H}, \mathrm{C}-6-\mathrm{H}) .\end{array}$ \\
\hline
\end{tabular}


Table 3. Anti-inflammatory activity of the compounds (1-8).

\begin{tabular}{ccccccc}
\hline \multirow{2}{*}{ Compound } & \multicolumn{5}{c}{ \% increase in paw thickness of various time intervals } \\
\cline { 2 - 7 } Standard & $0.5 \mathrm{~h}$ & $01 \mathrm{~h}$ & $2 \mathrm{~h}$ & $3 \mathrm{~h}$ & $4 \mathrm{~h}$ & $6 \mathrm{~h}$ \\
& $20.26 \pm$ & $23.95 \pm$ & $58.02 \pm$ & $67.93 \pm$ & $97.09 \pm$ & $98.98 \pm$ \\
Control & 0.64 & 0.66 & 1.54 & 1.65 & 1.95 & 1.98 \\
$\mathbf{1}$ & $1.59 \pm$ & 22.69 & 56.46 & 72.02 & 86.43 & $87.66 \pm$ \\
$\mathbf{2}$ & $36.97 \pm$ & \pm 1.71 & \pm 1.28 & \pm 1.86 & \pm 4.03 & 1.55 \\
& 0.78 & $3.54 \pm$ & $56.95 \pm$ & $65.22 \pm$ & $74.74 \pm$ & $87.66 \pm$ \\
$\mathbf{3}$ & $49.39 \pm$ & $50.60 \pm$ & $59.54^{* *}$ & 1.59 & 1.73 & 1.55 \\
& $2.97^{*}$ & $2.51^{*}$ & 0.96 & $74.90 \pm$ & $82.42 \pm$ & $94.43 \pm$ \\
$\mathbf{4}$ & $44.46 \pm$ & $69.62 \pm$ & $69.21 \pm$ & $78.66 \pm$ & $85.68 \pm$ & $97.05 \pm$ \\
& 2.20 & 1.01 & $2.58^{* *}$ & $4.77^{* * *}$ & $4.31^{* * *}$ & $4.32^{* *}$ \\
$\mathbf{5}$ & $36.79 \pm$ & $50.70 \pm$ & $64.55 \pm$ & $75.15 \pm$ & $88.36 \pm$ & $94.61 \pm$ \\
& $2.54^{*}$ & $3.77^{* *}$ & $3.28^{* *}$ & $3.21^{* *}$ & $3.31^{* *}$ & 1.95 \\
$\mathbf{6}$ & $36.26 \pm$ & $50.43 \pm$ & $55.27 \pm$ & $78.19 \pm$ & $82.24 \pm$ & $89.23 \pm$ \\
& $2.48^{*}$ & 1.36 & 1.44 & $4.47^{* * *}$ & $3.38^{* *}$ & $3.90^{* *}$ \\
$\mathbf{7}$ & $43.91 \pm$ & $60.66 \pm$ & $66.96 \pm$ & $78.91 \pm$ & $85.51 \pm$ & $94.55 \pm$ \\
& 1.80 & $2.92^{*}$ & $4.39^{* * *}$ & $4.79^{* * *}$ & $4.41^{* * *}$ & 2.04 \\
$\mathbf{8}$ & $18.64 \pm$ & $41.30 \pm$ & $41.88 \pm$ & $67.63 \pm$ & $76.32 \pm$ & $89.23 \pm$ \\
& 2.01 & $4.07^{* *}$ & 0.09 & 1.42 & $3.89^{* *}$ & $3.89^{* * *}$ \\
\hline
\end{tabular}

Control: 1\% sodium CMC gel, standard : aceclofenac standard and sample solution is $100 \mathrm{mg} / \mathrm{kg}$ body weight, values are expressed as mean $\pm(n=6) *=2.28, * *=3.75, * * *=4.35$.

$P^{*}<0.05, P^{* *}<0.01, P^{* * *}<0.001$ compared to control student $t$-test

\section{Results and Discussion}

The screening results revealed that the compounds (1-8) exhibited moderate to considerable activity when compared with reference standard aceclofenac. The synthesized compounds showed anti-inflammatory activity in the range of 50-80\% whereas standard drug showed $80-85 \%$ inhibition in paw edema. The results of anti-inflammatory activity indicated that compound $\mathbf{4}$ showed maximum anti-inflammatory activity.

\section{Acknowledgment}

We are thankful to the Head, Sophisticated Instrumentation Facility, Indian Institute of Sciences, Bangalore for ${ }^{1} \mathrm{H}$ NMR spectra and to Sipra Laboratories, Hyderabad for IR spectra.

\section{References}

1. Li R, Kenyon G L, Cohen F E, Chen X, Gong B, Domingeuz J N, Davidson E, Kurzban G, Miller R E, Nazum E O, Rosenthal P J and Meckewow J H, J Med Chem., 1995, 38, 5031.

2. Ballesteros J F, Sanz M J, Ubeda A, Miranda M A, Iborra S, Paya M and Alcaraz M J, J Med Chem., 1995, 38, 2794.

3. Yit C C and Das N P, Cancer Lett., 1994, 82, 65.

4. Satomi Y, Int J Cancer, 1993, 55, 506.

5. Wattenberg L W, Coccia J B and Galhaith A R, Cancer Lett., 1994, 83, 165.

6. Dinkova-Kostova A T, Abeygunawardana C and Talalay P, J Med Chem., 1998, 41, 5287.

7. Edwards M L, Stemerick D M and Sunkara P S, J Med Chem., 1990, 33, 1948.

8. Rajendra Prasad Y, Ravi Kumar P, Asha Deepti Ch and Venkata Ramana M, Asian J Chem., 2007, 19(6), 4799.

9. Singh H and Ghosh M N, J Pharm Pharmcol., 1958, 20, 316. 


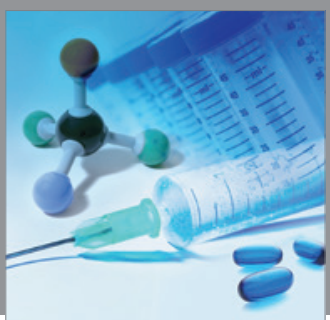

International Journal of

Medicinal Chemistry

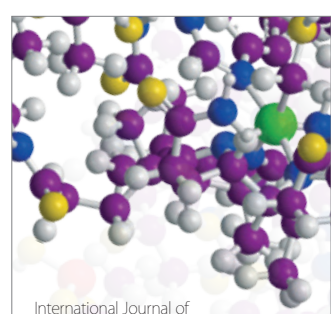

Carbohydrate Chemistry

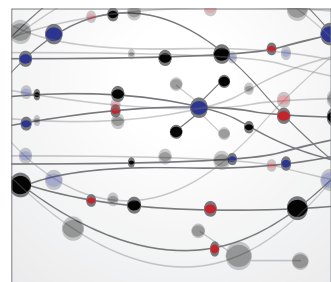

The Scientific World Journal
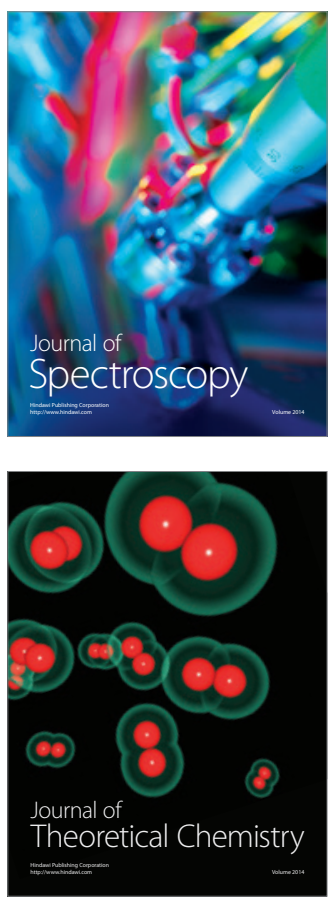
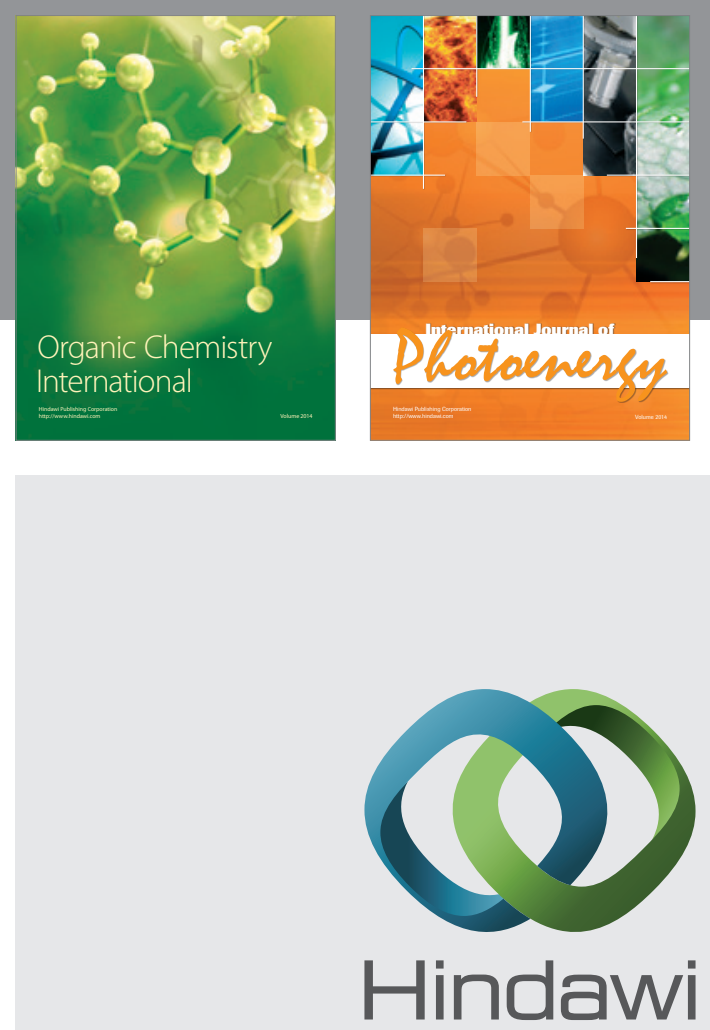

Submit your manuscripts at

http://www.hindawi.com
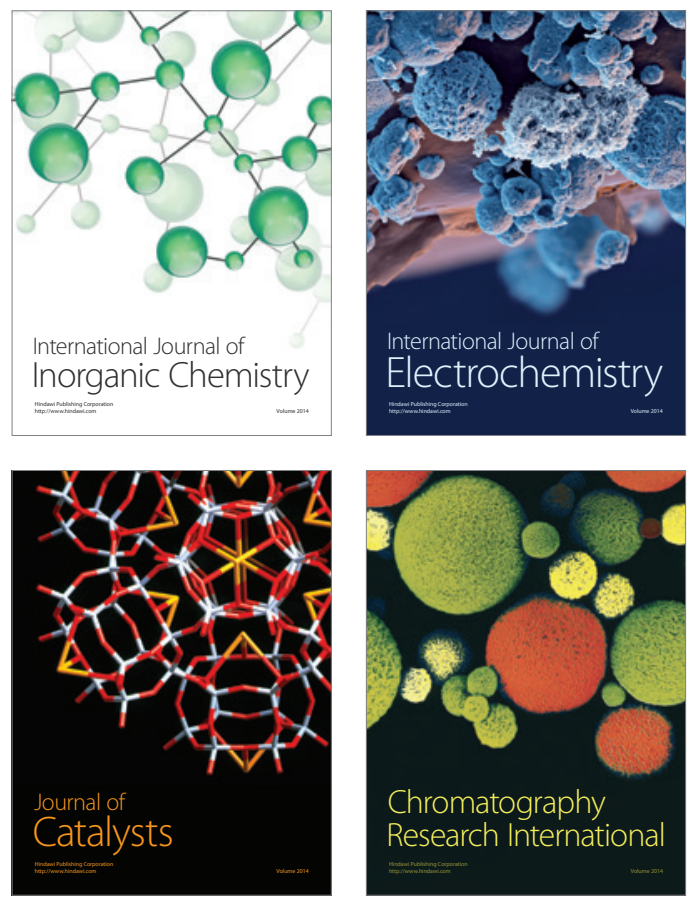
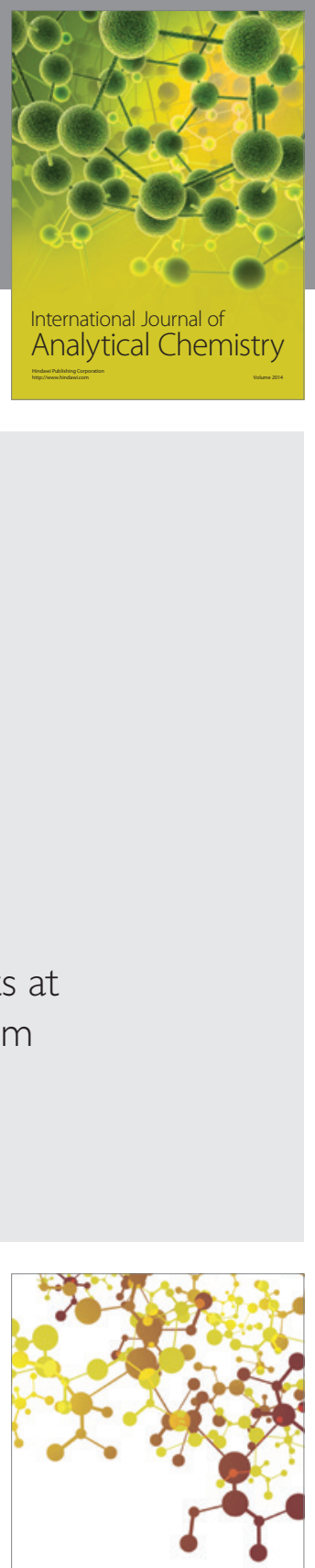

Journal of

Applied Chemistry
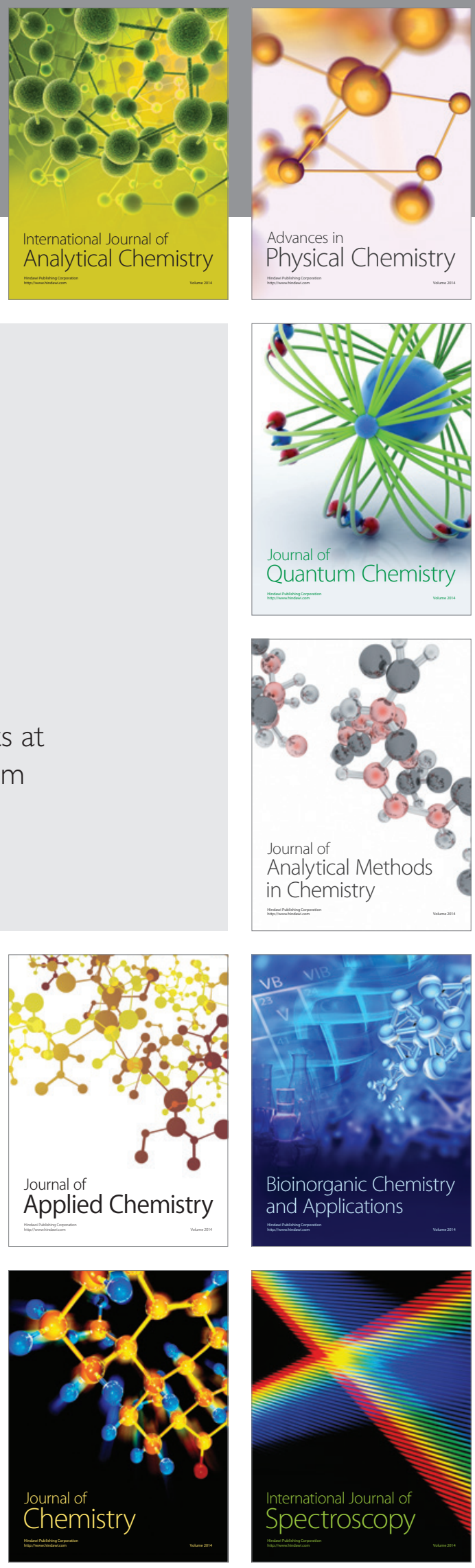